\title{
Response of pregnancy related functional constipation to acupressure in postpartum women
}

\author{
Marwa Abd-El Rahman $\mathrm{M}^{1 *}$, Azza Barmoud Nashed ${ }^{1}$, Mona Mohamed Taha² and Amir A. Gabr ${ }^{3}$
}

${ }^{*}$ Correspondence: dr.marwa2010@gmail.com

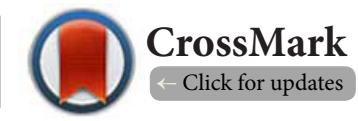

\begin{abstract}
'Assistant prof. in Department of Physical Therapy for woman's health, Faculty of Physical Therapy, Cairo University, Egypt. ${ }^{2}$ Assistant prof. in Department of Physical Therapy For Cardiovascular/Respiratory Disorder and Geriatrics, Faculty of Physical Therapy, Cairo University, Egypt.

${ }^{3}$ Assistant Prof. of Obstetrics and Gynecology, Faculty of Medicine, Cairo University.
\end{abstract}

\begin{abstract}
The aim of this study was to determine the effect of acupressure on pregnancy related functional constipation inpostpartum women, 40 post- partum primiparous women in 2 nd to 6 th week, after vaginal delivery with mediolateralepisiotomy participated in this study, they all complained from pregnancy related functional constipation diagnosed by Rome III criteria and confirmed by obstetrician, their ages were ranged from 24 to 35 years, their body mass index (BMI) was ranged from 25.5 to $33 \mathrm{~kg} /$ $\mathrm{m} 2$, they were randomly divided into 2 groups equal in number, (Group A) (Acupressure group) received acupressure therapy for 45 minutes in addition to30 min of morning walking on fresh air as well as general bowel care advice, (Group B) (Control group) received the same intervention as in (Group A) except for acupressure therapy program. All women in both groupswere evaluated before andafter 6 consecutive days of therapy;both groups recorded a statistical significant improvement in Patient Assessment ConstipationQuality of Life(PAC-QOL), Patient Assessment Constipation-Symptom(PAC-SYM) when compared with their corresponding values measured at pre-treatment. But (Group A) showed more improvement in all variables than (Group B).Finally it can be concluded thatAcupressure and walking with general bowel care advice lead to increase quality of life (QOL) and decrease severity of pregnancy related functional constipation symptoms in postpartum period.
\end{abstract}

Keywords: Post-Partum Period, Constipation and Acupressure

\section{Introduction}

Constipation is considered as one of the common medical problem that affect people all over the world, main manifestations of constipation arereduced defecation, lumpy or hard stools and straining, these symptoms considered as functional constipation when it does not have an anatomical or physiological cause. Also, it may affected by many factors as age, gender, socioeconomic status, dietary habits, education level, anxiety, depression,pregnancy and other psychological factors are associated with the prevalence of functional constipation. It is a common intestinal disease, especially found in elderly, pregnant and puerperal females [1].

Puerperium or postpartum period is a period starts about an hour after descent of the placenta and includes the following six weeks, if it lasts up to 6 months it called delayed postpartum period [2].

The postpartum period is a critical time for the new mother, new born and the whole family. Many problems may occur at this period that may cause physical illness and poor quality of life for both mother and her baby, in terms of morbidity and cost of treatment,constipation has a significant negative impact on the quality of life [3].

Females may suffer from constipation up to three to six months after labour and it may persist up to 12 months following labour [4].

Constipation is a common problem during the postpartum period for a many reasons asbowel tone and gastric motility, which were decreased during pregnancy due to excess progester- 
one, remains sluggish for a long time. In addition, relaxation of the abdominal muscles increases constipation and distension with gas. Restricted food and fluid intake during labour often results in small, hard stools. Perineal trauma, episiotomy, and haemorrhoids cause discomfort and interfere with effective bowel elimination. Many women suffer from pain when they attempt to pass stool and are unwilling to exert pressure on the perineum as well, taking iron supplementation is considered as an added cause of constipation [5].

Pharmacological interventions, laxatives are the drugs of choice toeliminate symptoms of constipation and can be taken orally in many forms either liquid, tablet, powder or granule form. Laxatives are grouped into: bulk forming laxatives, osmotic laxatives, stimulant laxatives, faecal softeners and lubricants [6].

Using laxatives and stool softeners for prolonged time have been associated with many significant clinical problems, including diarrhea, hypomagnesaemia, life-threatening hyper phosphatemia, hypo-albuminemia, an increased risk of faecal incontinence, and poor response to bowel preparation for barium enema [7].

Non-pharmacological interventions, diet adjustment that include: prober amount of fibre (such as fruits, vegetables, for example cucumber, and soup), water and fluids can help inalleviating symptoms and prevent recurrences of constipation. Fibres assoluble fibres (which helps soften the stools) and insoluble fibre (which adds bulk to the stools) both promote regular bowel movements [8].

Physical activity should be encouraged to be resumed as soon as the mother is able to do. This time point varies from one individual to another with some women able to return to routinephysical activity within days of delivery [4].

Acupressure is commonly used in some cultures and countries to manage many symptoms during pregnancy and after labour. It is a treatment based on traditional Chinese medicine and has the same principle as acupuncture. This technique is non-invasive and may prove to be a useful adjunct treatment in the care of a wide variety of complains [9].

Acupressure has a role in improving bowel function especially constipation, as it can promote gastrointestinal motility ,modulate the intestinal function ,stimulate the sacral nerve which can promote colonic motility, and improve pelvic floor awareness [10].

The Patient Assessment of Constipation-Quality of Life (PAC$\mathrm{QOL}$ ) questionnaire is considered as a universal assessment tool with reliability and validity in patient with constipation, measures the effect of constipation on daily life, it comprised of 28 items with four subscales such as Physical Discomfort, Psychosocial Discomfort, Worries and Concerns, and Satisfaction. The PAC-QOL consists of a 5-point Likert-type scale, and a lower score indicates higher or better quality of life [11].

The Patient Assessment of Constipation-Symptoms (PACSYM) questionnaire is frequently used in clinical trials of constipation. Itwasconstructed as an important tool for assessing the severity of patient-reported symptoms of constipation. It is considered as valid, responsive to change and provides a comprehensive means to evaluate the effectiveness of treatment for such cases [12].

So this study provided an insight for the benefits of the acupressure and its effect onmanaging pregnancy related functional constipation for postpartum women.

\section{Participants}

Forty postpartum primiparous women, (2-6 weeks) after vaginal delivery withmediolateral episiotomy participated in this study, their age and BMI were ranged between 26 to 35 years and 25.5 to $33 \mathrm{~kg} / \mathrm{m}^{2}$ respectively, they were all met Rome III criteria for functional constipation, namely, they should suffer in the last 3 months from at least two or more of the following criteria: straining inabout $25 \%$ of defecations; lumpy or hard stools in about $25 \%$ of defecations; sensation of incomplete evacuation in about $25 \%$ of defecations; sensation of anorectal obstruction/blockage in about $25 \%$ of defecations; manual maneuvers to facilitate about $25 \%$ of defecations (e.g., manual evacuation, support of the perineum ); and fewer than three defecations per week [13].

They were all recruited from outpatient clinic of gynaecology and obstetrics, Kasr Al Aini teaching hospital, all participants provided written informed consent. The study was carried out from May to November 2017 and was approved by ethical committee of Faculty of Physical Therapy Cairo University No: P.T.REC/012/001792.

Women who had history of inflammatory bowel disease, anal fissure andhistory of bowel surgery (other than appendectomy) as well as endocrine disease or digestive tract disease, irritable bowel syndrome and history of genital prolapse were excluded from this study.

\section{Study design}

The study was a randomized, parallel group trial.

\section{Primary Outcome Measures}

The primary outcome measure was patient assessment of constipation-related health quality of life by means of the Patient Assessment of Constipation Quality of Life Questionnaire (PAC-QOL). While secondary outcome was patient assessment constipation- symptom (PAC-SYM) distribution.

\section{Sample size estimation}

Based on pilot study, 10 subjects/group, sample size was calculated, Assuming $a=0.05$, an effect size $=0.41$ and power of $80 \%$, so a sample size of 20 subjects per group would be required (GPower 301 http:www.psycho.uniduesseldorf.de).

\section{Randomization}

Simplerandomization was performed by asking the patient to randomly choose a piece of paper with (A) or (B) letter. So participants were randomly assigned into one of the two groups. 
Group (A) (Acupressure group)

Consisted of twenty women who received morning acupressure for 45 minutes in addition to morning walking in fresh air for $30 \mathrm{~min}$. for six consecutive days as well as general bowel care advices.

\section{Group (B) (Control group)}

Consisted of twenty women receivedmorning walking in fresh air for 30 mininstructed to follow general bowel care advices- as in group (A) -which are:

1. Encourage adequate fibre intake (20 to 35 grams each day(.

2. Avoid caffeine-containing foods and beverages.

3. Drink at least 1.5 and 2.0 Litre of water/ day.

4. Proper defecation position:

The correct position can improve the angle of the rectum, help muscles to work efficiently and prevent straining, so:

Sit with feet apart and resting on blocks or stool, relaxthe abdominal muscles, lean forward and place either hands or forearms on the thighs, do not sit for longer than ten minutes -If your bowels have not pass try again later- maintain the same bathroom schedule every day, 20 minutes before breakfast (even if you can't have a bowel movement, still go through the actions) [14].

5. Provide support during a bowel movement by pressing a fresh sanitary pad against perineum, holding it while defecate to reducethe pain and discomfort.

\section{Evaluative procedures}

Assessment of constipation pre and post study was done through:

\section{a. Patient Assessment of Constipation Quality of Life Questionnaire (PAC-QOL)}

The validated PAC-QOL was composed of 28 items classified into 4 subscales: physicaldiscomfort and psychosocial discomfort, in addition to worries and concerns and finally satisfaction. The first three subscales comprise the patient dissatisfaction index (where higher great corresponded to worse quality of life). The satisfaction subscale includes 4items (where higher great corresponded to improve quality of life) [11].

- Every woman in both groups (A, B) was asked to answer every question after reading and explaining each one.

\section{b. Patient Assessment of Constipation-Symptom(PAC- SYM)}

It was constructed to assess symptom frequency and severity of constipation. This 12 -item self-report measure was divided into three symptom subscales (i.e. abdominal, rectal and stool). Items were scored on a four-point scale, with 4 grades where higher scores indicating the worst symptom severity [15].

\section{Treatment procedure}

Before starting the first treatment session, each patient was instructed briefly about the treatment procedure and how to make it as a home routine, which was explained carefully to her to gain her confidence and cooperation.

\section{Acupressure program}

Morning application of thumb moderate perpendicular pressurewas done for $5 \mathrm{~min}$, interspersed by 5 second of release for 5 second on the following acupressure points:

\section{Stomach 25 (ST25)}

Called Tianshu, it is located on the abdomen, 2 cun (about 3 fingers) Lateral to the umbilicus.

\section{Spleen 14 (SP14)}

Called Fujie, it is located on the lower abdomen, 3 fingers down from SP 15 which is located on 4 fingers lateral to umbilicus).

\section{Large intestine 11 (LI11)}

Called Quchi, it is located on elbow, when the elbow is flexed the point lies in the depression at the lateral end of the transverse cubital crease, midway between LU5(which is located in the depression at the radial side of the tendon of biceps brachii )and the lateral epicondyle of the humerus (done bilaterally).

\section{Stomach 36 (ST36)}

Called $\mathrm{Zu}$ San $\mathrm{Li}$, it is located on the anterior aspect of the lower leg, 3 cun (about 4 fingers) below the inferior border of the Patella one finger width lateral to the edge of the tibia, there is a small notch next to the tibia at this point ( done bilaterally).

\section{large intestine 4(LI4)}

Called Hegu, it is located on the dorsum of the hand, between the 1st and 2nd metacarpal bones, in the middle of the $2^{\text {nd }}$ metacarpal bone on the radial side (done bilaterally).

\section{Data Analysis and Statistical Design}

The data were collected before and after treatment, the collected data was statistically analysed using:

a. Descriptive statistics in the form of:

- The mean $(X)$, and the standard deviation (SD).

- Both (The mean and the standard deviation) of each group were calculated for each parameter.

- $\quad \operatorname{Mean}(x)=$ summation of $x /$ number of $x$.

- Standard deviation (SD) =root square of variance.

\section{Inferential statistics in the form of:}

- Unpaired t test: was used to compare data between groups for parametric data (age, weight and height).

- Chi-Square test and Wilcoxon Test: were carried out to examine the differences between and within the two groups before and after the treatment for non-parametric data. - P-value: is the degree of significance, which is selected at 
$5 \%$ level. P-value $>0.05$ indicates non significance results, while $\mathrm{P}$-value $<0.001$ indicates highly significance results.

\section{Results}

Demographic data of both groups

There were no statistical significant differences between the mean values for both groups regarding age, weight, height and BMI pre-treatment.

Patient Assessment Constipation-Quality of Life(PACQOL) questionnaire

\section{Physical discomfort distribution}

Results of Physical discomfort distribution within groups analyzed by using Wilcoxon test to show difference between physical discomfort distributions pre and post treatment for both groups which was statistically very highly significant as the $z$-value $=-3.794$ in group $a$ and 3.500in group b, $p$-value $=0.001$ for both groups, (Table 1).

While physical discomfort distribution between groups was analyzed by Chi square test to show difference between physical discomfort distributions pre study which revealed non-significant difference as $\chi 2=1.000$ and $p$-value $=0.607$ while there was statistical very highly significant difference in favor to the study group (A) with chi square test $=26.222$ and $p$-value $=0.001$, (Table 2$)$.

\section{Psychosocial discomfort distribution}

Psychosocial discomfort distribution within groups was analyzed by wilcoxon test to show difference between psychosocial discomfort distributions pre-treatment and post study in both groups (A) and (B) revealing astatistical very highly significant results as the $z$-value $=-3.695$ and -3.207 respectively and p-value was 0.001 for both groups, (Table 3 ).

While psychosocial discomfort distribution between groups was evaluated by using Chi square to show difference between psychosocial discomfort distributions pre-treatment between the two groups Which revealed a statistical non-significant results as $\chi 2=1.000$ and $p$-value $=0.607$, While post treatment there was astatistical very highly significant with $\chi 2=27.583$ and $p$-value $=0.001$, (Table 4).

\section{Worries and concerns distribution}

Wilcoxon test was used to show difference between worries and concerns distributions pre and post study for both groups

Table 1. Physical discomfort distribution within the two groups.

\begin{tabular}{llllllllllll}
\hline & \multicolumn{3}{c}{ Pre-study } & & \multicolumn{7}{c}{ Post- study } \\
\hline & & $\mathbf{0}$ & $\mathbf{1}$ & $\mathbf{2}$ & $\mathbf{3}$ & $\mathbf{4}$ & $\mathbf{0}$ & $\mathbf{1}$ & $\mathbf{2}$ & $\mathbf{3}$ & $\mathbf{4}$ \\
\hline Group (A) & Frequency & 0 & 0 & 4 & 11 & 5 & 9 & 8 & 1 & 2 & 0 \\
& Percentage & $0 \%$ & $0 \%$ & $20 \%$ & $55 \%$ & $25 \%$ & $45.0 \%$ & $40 \%$ & $5 \%$ & $10 \%$ & $0 \%$ \\
\hline \multirow{2}{*}{ Group (B) } & Frequency & 0 & 0 & 2 & 11 & 7 & 0 & 1 & 8 & 10 & 1 \\
& Percentage & $0 \%$ & $0 \%$ & $10 \%$ & $55 \%$ & $35 \%$ & $0 \%:$ & $5 \%$ & $40 \%$ & $50 \%$ & $5 \%$ \\
\hline
\end{tabular}

Table 2. Physical discomfort distribution pre and post-treatment between the two groups.

\begin{tabular}{lllllllllll}
\hline grade & \multicolumn{3}{l}{ group (A) } & \multicolumn{3}{c}{$(\mathbf{n}=\mathbf{2 0})$} & \multicolumn{4}{c}{ Group (B) (n=20 ) } \\
\cline { 2 - 12 } & $\mathbf{0}$ & $\mathbf{1}$ & $\mathbf{2}$ & $\mathbf{3}$ & $\mathbf{4}$ & $\mathbf{0}$ & $\mathbf{1}$ & $\mathbf{2}$ & $\mathbf{3}$ & $\mathbf{4}$ \\
\hline Pre study & $0 \%$ & $0 \%$ & $20 \%$ & $55 \%$ & $25 \%$ & $0 \%$ & $0 \%$ & $10 \%$ & $55 \%$ & $35 \%$ \\
Post study & $45 \%$ & $40 \%$ & $5 \%$ & $10 \%$ & $0 \%$ & $0 \%$ & $5 \%$ & $40 \%$ & $50 \%$ & $5 \%$ \\
\hline
\end{tabular}

Table 3. Psychosocial discomfort distribution within the two groups.

\begin{tabular}{llllllllllll}
\hline & grade & \multicolumn{1}{c}{ Pre } & \multicolumn{7}{c}{ Post } \\
\cline { 3 - 12 } & & $\mathbf{0}$ & $\mathbf{1}$ & $\mathbf{2}$ & $\mathbf{3}$ & $\mathbf{4}$ & $\mathbf{0}$ & $\mathbf{1}$ & $\mathbf{2}$ & $\mathbf{3}$ & $\mathbf{4}$ \\
\hline Group (A) & Frequency & 0 & 0 & 5 & 11 & 4 & 12 & 5 & 1 & 2 & 0 \\
& Percentage & $0 \%$ & $0 \%$ & $25 \%$ & $55 \%$ & $20 \%$ & $60 \%$ & $25 \%$ & $5 \%$ & $10 \%$ & $0 \%$ \\
\hline Group (B) & Frequency & 0 & 0 & 7 & 11 & 2 & 0 & 1 & 15 & 4 & 0 \\
& Percentage & $0 \%$ & $0 \%$ & $35 \%$ & $55 \%$ & $10 \%$ & $0 \%:$ & $5 \%$ & $75 \%$ & $20 \%$ & $0 \%$ \\
\hline
\end{tabular}

Table 4. Psychosocial discomfort distribution between the two groups.

\begin{tabular}{lllllllllll}
\hline grade & \multicolumn{3}{c}{ Group (A) } & $(\mathbf{n}=\mathbf{2 0})$ & \multicolumn{4}{c}{ Group (B) $(\mathbf{n}=\mathbf{2 0})$} \\
\cline { 2 - 11 } & $\mathbf{0}$ & $\mathbf{1}$ & $\mathbf{2}$ & $\mathbf{3}$ & $\mathbf{4}$ & $\mathbf{0}$ & $\mathbf{1}$ & $\mathbf{2}$ & $\mathbf{3}$ & $\mathbf{4}$ \\
\hline Pre study & $0 \%$ & $0 \%$ & $25 \%$ & $55 \%$ & $20 \%$ & $0 \%$ & $0 \%$ & $35 \%$ & $55 \%$ & $10 \%$ \\
\hline Post study & $60 \%$ & $25 \%$ & $5 \%$ & $10 \%$ & $0 \%$ & $0 \%$ & $5 \%$ & $75 \%$ & $20 \%$ & $0 \%$ \\
\hline
\end{tabular}


$A$ and $B$ revealing very highly statistical significant difference as the $z$-value $=-3.779$ and -2.887 respectively, and $p$-value $=0.001$, (Table 5).

\section{Worries and concerns distribution between groups}

Chi square test was used to show difference between worries and concerns distributions pre-treatment between the two groups revealing statistically non-significant with chi square test $=2.643$ and $p$-value $=0.267$. While post-study there was statistically very highly significant difference with chi square test $=25.086$ and $p$-value $=0.001$, (Table 6).

Satisfaction distribution, Chi square test was used to show difference between satisfaction distribution post treatments between the two groups. There was statistically very highly significant difference with chi square test $=21.104$ and $p$-value $=0.001$, (Table 7).
Patient Assessment Constipation- Symptom (PAC-SYM) distribution

In group A(study group),Wilcoxon test was used to show difference between PAC-SYM distributions, pre-treatment and post treatment revealing a statistically very highly significant as the $z$-value $=-3.808$ and $p$-value $=0.001$, while In the group $\mathrm{B}$ (control group) pre -study and post treatment revealing a statistically significant difference as the $z$-value $=-2.828$ and p-value $=0.005$, (Table 8).

Between groups, Chi square test was used to show difference between PAC-SYM distributions pre -treatment between the two groups revealing statistically significant difference with chi square test $=5.227$ and $p$-value $=0.022$, While post treatment between the two groups results revealing statistically very highly significant difference with chi square test $=22.222$ and $p$-value $=0.001$, (Table 9).

Table 5. Worries and concerns distribution within the two groups.

\begin{tabular}{llllllllllll}
\hline & Grade & \multicolumn{1}{l}{ Pre study } & \multicolumn{1}{c}{ Post study } \\
\cline { 3 - 12 } & & $\mathbf{0}$ & $\mathbf{1}$ & $\mathbf{2}$ & $\mathbf{3}$ & $\mathbf{4}$ & $\mathbf{0}$ & $\mathbf{1}$ & $\mathbf{2}$ & $\mathbf{3}$ & $\mathbf{4}$ \\
\hline Group (A) & Frequency & 0 & 0 & 6 & 12 & 2 & 11 & 6 & 1 & 1 & 1 \\
& Percentage & $0 \%$ & $0 \%$ & $30 \%$ & $60 \%$ & $10 \%$ & $55 \%$ & $30 \%$ & $5 \%$ & $5 \%$ & $5 \%$ \\
\hline Group (B) & Frequency & 0 & 0 & 9 & 11 & 0 & 0 & 3 & 13 & 4 & 0 \\
& Percentage & $0 \%$ & $0 \%$ & $45 \%$ & $55 \%$ & $0 \%$ & $0 \%$ & $15 \%$ & $65 \%$ & $20 \%$ & $0 \%$ \\
\hline
\end{tabular}

Table 6. Worries and concerns distribution between the two groups.

\begin{tabular}{lllllllllll}
\hline grade & \multicolumn{3}{c}{ group (A) } & \multicolumn{2}{c}{$(\mathbf{n}=\mathbf{2 0})$} & \multicolumn{6}{c}{ Group (B) $(\mathbf{n = 2 0})$} \\
\cline { 2 - 12 } & $\mathbf{0}$ & $\mathbf{1}$ & $\mathbf{2}$ & $\mathbf{3}$ & $\mathbf{4}$ & $\mathbf{0}$ & $\mathbf{1}$ & $\mathbf{2}$ & $\mathbf{3}$ & $\mathbf{4}$ \\
\hline Pre study & $0 \%$ & $0 \%$ & $30 \%$ & $60 \%$ & $10 \%$ & $0 \%$ & $0 \%$ & $45 \%$ & $55 \%$ & $0 \%$ \\
\hline Post study & $55 \%$ & $30 \%$ & $5 \%$ & $5 \%$ & $5 \%$ & $0 \%$ & $15 \%$ & $65 \%$ & $20 \%$ & $0 \%$ \\
\hline
\end{tabular}

Table 7. Satisfaction distribution post treatment between the two groups.

\begin{tabular}{lllllllllll}
\hline grade & \multicolumn{3}{c}{ group (A) } & \multicolumn{2}{c}{$(\mathbf{n}=\mathbf{2 0})$} & \multicolumn{4}{c}{ Group (B) $(\mathbf{n}=\mathbf{2 0})$} \\
\cline { 2 - 12 } & $\mathbf{0}$ & $\mathbf{1}$ & $\mathbf{2}$ & $\mathbf{3}$ & $\mathbf{4}$ & $\mathbf{0}$ & $\mathbf{1}$ & $\mathbf{2}$ & $\mathbf{3}$ & $\mathbf{4}$ \\
\hline Post study & $5 \%$ & $10 \%$ & $0 \%$ & $33 \%$ & $50 \%$ & $15 \%$ & $25 \%$ & $40 \%$ & $20 \%$ & $0 \%$ \\
\hline
\end{tabular}

Table 8. PAC-SYM distribution within the two groups (A\&B).

\begin{tabular}{llllllllllll}
\hline & Grade & \multicolumn{1}{l}{ Pre } & \multicolumn{1}{c}{ post } \\
\cline { 3 - 12 } & & $\mathbf{0}$ & $\mathbf{1}$ & $\mathbf{2}$ & $\mathbf{3}$ & $\mathbf{4}$ & $\mathbf{0}$ & $\mathbf{1}$ & $\mathbf{2}$ & $\mathbf{3}$ & $\mathbf{4}$ \\
\hline Group (A) & Frequency & 0 & 0 & 8 & 12 & 0 & 9 & 8 & 2 & 1 & 0 \\
& Percentage & $0 \%$ & $0 \%$ & $40 \%$ & $60 \%$ & $0 \%$ & $45 \%$ & $40 \%$ & $10 \%$ & $5 \%$ & $0 \%$ \\
\hline Group (B) & Frequency & 0 & 0 & 16 & 4 & 0 & 0 & 4 & 16 & 0 & 0 \\
& Percentage & $0 \%$ & $0 \%$ & $80 \%$ & $20 \%$ & $0 \%$ & $0 \%$ & $20 \%$ & $80 \%$ & $0 \%$ & $0 \%$ \\
\hline
\end{tabular}

Table 9. PAC-SYM distribution between the two groups.

\begin{tabular}{lllllllllll}
\hline grade & \multicolumn{3}{c}{ group (A) } & $(\mathbf{n}=\mathbf{2 0})$ & \multicolumn{4}{c}{ Group (B) $(\mathbf{n = 2 0})$} \\
\cline { 2 - 11 } & $\mathbf{0}$ & $\mathbf{1}$ & $\mathbf{2}$ & $\mathbf{3}$ & $\mathbf{4}$ & $\mathbf{0}$ & $\mathbf{1}$ & $\mathbf{2}$ & $\mathbf{3}$ & $\mathbf{4}$ \\
\hline Pre study & $0 \%$ & $0 \%$ & $40 \%$ & $60 \%$ & $0 \%$ & $0 \%$ & $0 \%$ & $80 \%$ & $20 \%$ & $0 \%$ \\
\hline Post study & $45 \%$ & $40 \%$ & $10 \%$ & $5 \%$ & $0 \%$ & $0 \%$ & $20 \%$ & $80 \%$ & $0 \%$ & $0 \%$ \\
\hline
\end{tabular}


Abd-El Rahman et al, Physical Therapy and Rehabilitation 2018,

http://www.hoajonline.com/journals/pdf/2055-2386-5-11.pdf

doi: 10.7243/2055-2386-5-11

\section{Discussion}

The obtained results of this study were agreed with Eun., (2010) [9] who assessed the effectiveness of acupressure on constipation, quality of life affection and depressive symptoms in cancer patients with constipation, and reported that acupressure at SP 14 for 5 minutes, daily for 7 days was effective in decreasing constipation, so acupressure could be a safe and cost-effective alternative therapy for constipation in cancer patients.

Acupressure can improve symptoms of constipation in stroke patients (Wang, 2006) [16], and neurological disorders (Chen, 2006) [17].

The result of this study was in agreement with Hui-Lin et al.,(2013) [18], who evaluated the effectiveness of ST-36 (Zusanli) acupressure on recovery of postoperative gastrointestinal function in patients with colorectal cancer and reported that application of ST-36 acupressure for five consecutive days, can improve Gl function postoperativelyin patients with colorectal cancer by improving the frequency of bowel sounds and decreasing the time to first flatus passage and first liquid food intake.

It was observed that a lower level of motilin was noted in patients of functional constipation and found to be elevated with acupuncture at ST36 and ST37. Also, acupuncture bilaterally at ST25 was reported to enlarge colonic smooth muscle thickness and number of Cajal cells considerably. Vagal and parasympathetic mechanisms also have an impact on the accelerative effect of acupuncture on colon motility [19].

The obtained results of this study were also agreed with Wai et al., (2015) [20] who practiced acupressure for 10 days in addition to abdominal massage in patients with constipation, comparing to control group he found improvement in symptom severity and perceived quality of life immediately and 2 weeks after completion of the intervention.

The result of this study was in agreement with Jung et al., (2008) [21] who assessed the effectiveness of meridian acupressure on functional constipation in bed-ridden aged in patients, The results suggest that relief of constipation for bed-ridden aged inpatients was properly achieved by meridian acupressure. Patients with constipation can use the Meridian Acupressure as a non-invasive nursing intervention instead of a laxative or enemaneeds validation by using a follow -up research.

The obtained results of this study were also supported by Bai Tao et al., (2016) [22] who assessed the impact of acupuncture on the treatment of functional constipation and found that in comparison to medication, acupuncture was more effective and had a lower adverse effect rate and less harm in the treatment of constipation. A short course of treatment of two weeks was sufficient for good results. However, the low methodological quality, small sample size, unidentified risk of bias and various evaluation indexes of the trials included in this Meta-analysis indicates the need for large, multicentre randomized controlled trial (RCTs) assessing the effectiveness and safety of acupuncture in patients with constipation.

Our results were also agreed with Lee and Shon (2006) [23] who studied the effect of meridian acupressure on alleviating constipation severity in patients withstroke. And found that there was a significant improvement in frequency of defecation and decrease severity of constipation in the experimental group compared to the other control group.

This study was in disagreement with Klauser et al., (1993) [24] who investigated the effectiveness of body acupressure on stool frequency and colonic transit time in patient suffered from constipation and reported that frequencies of stool and colonic transit time were not significantly different in control and acupressuregroup,so acupressureas performed in this study does not influence objective parameters of colonic function to a clinically relevant degree.

\section{Conclusion}

Finally, it could be concluded that acupressure and walking in addition to general bowel care advice are significantly improve quality of life (QOL) and decrease severity of pregnancy related functional constipation symptoms in postpartum period.

\section{Competing interests}

The authors declare that they have no competing interests.

Authors' contributions

\begin{tabular}{|l|c|c|c|c|}
\hline Authors' contributions & MAR & ABN & MMT & AAG \\
\hline Research concept and design & $\checkmark$ & $\checkmark$ & $\checkmark$ & $\checkmark$ \\
\hline Collection and/or assembly of data & $\checkmark$ & $\checkmark$ & $\checkmark$ & -- \\
\hline Data analysis and interpretation & $\checkmark$ & $\checkmark$ & $\checkmark$ & -- \\
\hline Writing the article & $\checkmark$ & $\checkmark$ & $\checkmark$ & $\checkmark$ \\
\hline Critical revision of the article & $\checkmark$ & $\checkmark$ & $\checkmark$ & $\checkmark$ \\
\hline Final approval of article & $\checkmark$ & $\checkmark$ & $\checkmark$ & $\checkmark$ \\
\hline Statistical analysis & $\checkmark$ & $\checkmark$ & $\checkmark$ & $\checkmark$ \\
\hline
\end{tabular}

Acknowledgements

We acknowledge all patients who participated in our study.

Publication history

Editor: Mohammad H. Hadadzadeh,Wheeling Jesuit University, USA. Received: 23-May-2018 Final Revised: 30-Jun-2018

Accepted: 24-Jul-2018 Published: 17-Aug-2018

\section{References}

1. Shi W, Xu X, Zhang Y, Guo S and Wang J. Epidemiology and Risk Factors of Functional Constipation in Pregnant Women. PLoS One. 2015; 10:e0133521. | Article | PubMed Abstract | PubMed FullText

2. Romano M, Cacciatore A, Giordano R and La Rosa B. Postpartum period: three distinct but continuous phases. J Prenat Med. 2010; 4:22-5. I PubMed Abstract | PubMed FullText

3. Turawa EB, Musekiwa A and Rohwer AC. Interventions for treating postpartum constipation. Cochrane Database Syst Rev. 2014; CD010273. | Article | PubMed

4. Rungsiprakarn $P$, Malinee L, Ussanee S. Pisake L and Jeremy J. Interventions for treating constipation in pregnancy. Cochrane Database of Systematic Reviews. 2014; 12:1-7. 
Abd-El Rahman et al, Physical Therapy and Rehabilitation 2018, http://www.hoajonline.com/journals/pdf/2055-2386-5-11.pdf

5. Blackburn S. Maternal, fetal and neonatal physiology. A clinical perspective ( $3^{\text {rd }}$ Ed). St. Louis, Saunders. 2007; 350-370.

6. Candy B, Jones L, Goodman ML, Drake R and Tookman A. Laxatives or methylnaltrexone for the management of constipation in palliative care patients. Cochrane Database Syst Rev. 2011; CD003448. | Article | PubMed

7. Rao SS, Rattanakovit $\mathrm{K}$ and Patcharatrakul T. Diagnosis and management of chronic constipation in adults. Nat Rev Gastroenterol Hepatol. 2016; 13:295-305. | Article | PubMed

8. Balch P. A Practical A-to-Z Reference to Drug-free Remedies using Vitamins, Minerals, Herbs \& Food Supplements. $5^{\text {th }}$ Ed. Penguin New York. 2010; 220-225.

9. Eun Jin Lee. The effect of acupressure on constipation, quality of life, and depressive symptoms in cancer patients with constipation. University of Kentucky doctoral dissertations. 2010; 14:83-113. | Pdf

10. Shuqing D, Xun J, Yijiang D, Lingling W and Huifeng Z. Acupuncture in Treatment of Chronic Functional Constipation. Complementary and Alternative Medicine, 2015; 51:978-953.

11. Marquis P, De La Loge C, Dubois D, McDermott A and Chassany O. Development and validation of the Patient Assessment of Constipation Quality of Life questionnaire. Scand J Gastroenterol. 2005; 40:540-51. I Article I PubMed

12. Frank L, Kleinman L, Farup C, Taylor L and Miner P, Jr. Psychometric validation of a constipation symptom assessment questionnaire. Scand J Gastroenterol. 1999; 34:870-7. | PubMed

13. Drossman DA. The functional gastrointestinal disorders and the Rome III process. Gastroenterology. 2006; 130:1377-90. | Article | PubMed

14. Queensland Health. First steps in the management of urinary incontinence in community-dwelling older people. A clinical practice guideline. $2^{\text {nd }} \mathrm{Ed}$. 2007; 1-5.

15. Shruti S and Brij B. Scoring Systems in Evaluation of Constipation and Obstructed Defecation Syndrome (ODS). JIMSA. 2012; 25:57-59.

16. Wang HP, Horng HC, Lu CM, Chen MY, Chen HC, Chang $\mathrm{CH}$ and Lin $\mathrm{CH}$. The effectiveness of acupressure on stroke patient with constipation. Chang Gung Nurs. 2006; 17:418-27.

17. Chen $Y$, Chang $Y$ and Bai $C$. The effectiveness of acupressure at relieving constipation in neurological patients. J Evid Based Nurs. 2006; 2:301-10.

18. Chao HL, Miao SJ, Liu PF, Lee HH, Chen YM, Yao CT and Chou HL. The beneficial effect of ST-36 (Zusanli) acupressure on postoperative gastrointestinal function in patients with colorectal cancer. Oncol Nurs Forum. 2013; 40:E61-8. | Article | PubMed

19. Wang $X$ and Yin J. Complementary and Alternative Therapies for Chronic Constipation. Evid Based Complement Alternat Med. 2015; 2015:396396. | Article | PubMed Abstract | PubMed FullText

20. Wong WK, Chien WT and Lee WM. Self-administered acupressure for treating adult psychiatric patients with constipation: a randomized controlled trial. Chin Med. 2015; 10:32. | Article | PubMed Abstract | PubMed FullText

21. Jung, Young-Hoe, Jun and Jum-Yi. The Effect of Meridian Acupressure on Constipation in the Bed-ridden Aged with Stroke. Korean Journal of Adult Nursing. 2008; 20:664-673.

22. Bai T, Song $C$, Zheng $C$ and Huang $G$. Acupuncture for the treatment of functional constipation. J Tradit Chin Med. 2016; 36:578-87. I PubMed

23. Kang HS, Sok SR and Kang JS. Effects of Meridian acupressure for stroke patients in Korea. J Clin Nurs. 2009; 18:2145-52. I Article I PubMed

24. Klauser AG, Rubach A, Bertsche $O$ and Muller-Lissner SA. Body acupuncture: effect on colonic function in chronic constipation. $Z$ Gastroenterol. 1993; 31:605-8. | PubMed

\section{Citation:}

Abd-El Rahman M, Nashed AB, Taha MM and Gabr AA. Response of pregnancy related functional constipation to acupressure in postpartum women. Phys Ther Rehabil. 2018; 5:11.

http://dx.doi.org/10.7243/2055-2386-5-11 